\title{
Ultrafast High-Energy Electron Radiography Application in Magnetic Field Delicate Structure Measurement
}

\author{
J. H. Xiao, ${ }^{1,2}$ Y. C. Du $\mathbb{D}^{1,2}$ S. Z. Zhang, ${ }^{3}$ and Y. T. Zhao ${ }^{3}$ \\ ${ }^{1}$ Department of Engineering Physics, Tsinghua University, Beijing 100084, China \\ ${ }^{2}$ Key Laboratory of Particle \& Radiation Imaging (Tsinghua University), Ministry of Education, Beijing 100084, China \\ ${ }^{3}$ Xi'an Jiaotong University, Xi'an 710049, China
}

Correspondence should be addressed to Y. C. Du; dych@mail.tsinghua.edu.cn

Received 12 October 2020; Accepted 30 November 2020; Published 20 January 2021

Academic Editor: T. Shao

Copyright (C 2021 J. H. Xiao et al. This is an open access article distributed under the Creative Commons Attribution License, which permits unrestricted use, distribution, and reproduction in any medium, provided the original work is properly cited.

Transient electromagnetic field plays very important roles in the evolution of high-energy-density matter or laser plasma. Now, a new design is proposed in this paper to diagnose the transient magnetic field, using relativistic electron bunch as a probe based on high-energy electron radiography. And based on this scheme, the continuous distribution of magnetic strength field can be snapshotted. For $1 \mathrm{~mm}$ thick quadrupole magnet model measured by $50 \mathrm{MeV}$ probe electron beams, the simulation result indicates that this diagnosis has spatial resolution better than 4 microns and high measurement accuracy for strong magnetic strength and high magnetic gradient field no matter whether the magnetic interaction is focusing or defocusing for the range from $-510 \mathrm{~T}^{*} \mu \mathrm{m}$ to $510 \mathrm{~T}^{*} \mu \mathrm{m}$.

\section{Introduction}

The transient electromagnetic field plays very important roles in the research on the high-energy-density matter, driven by intense laser, ion, and $Z$-pinch equipment [1-6]. The research of electromagnetic field in plasma is related to accelerator physics, high-energy density physics, inertial confinement fusion, and astrophysics phenomena [7-12]. In the past several years, many methods based on charged particle radiography have been tried, such as laser wake field accelerator electron radiography, monoenergetic electron radiography, and monoenergetic proton radiography [13-19]. In this paper, we demonstrate a new method to achieve continuous point measurement for the electromagnetic field strength.

As a diagnosis probe, high-energy electron has some advantages for the transient electromagnetic field measuring. Relativistic speed beams are taken easily by making the electrons penetrate specimen with millimeter size in several tens of picoseconds; compared with the other relativistic particles, electron has lower magnetic rigidity, which makes it more sensitive to the electromagnetic field; besides, ultrashort bunch is taken easier, to ensure the quasistatic of diagnosed specimen.
High-energy electron radiography (HEER) has been developed at LANL, Tsinghua University and Institute of Modern Physics, Chinese Academy of Science, in the past several years [20-26]. The several microns spatial resolution has been also taken in the experiment. In the past years, only static target or fluid target has been used as the object in HEER experiment. Up to now, there is no report of using the point-to-point HEER as the electromagnetic field diagnosis. In this paper, a new method is proposed that is based on HEER beam line design for magnetic field area diagnosis.

\section{Principle}

Above all, two approximates can ensure the accuracy of this diagnosis. Firstly, the electric field is very similar to electrostatic field in the plasma. Thence, for the process from infinity to infinity, all the incident and exit potential of probe beams are zero, and there is no energy decrease or increase when the electron beam has penetrated the system. Secondly, for the scattered electron beams, the E/B field area is filled with very small amount of materials to intervene the electron scattering angle, just like the ultrashort intense laser-foil interaction process [19]. 


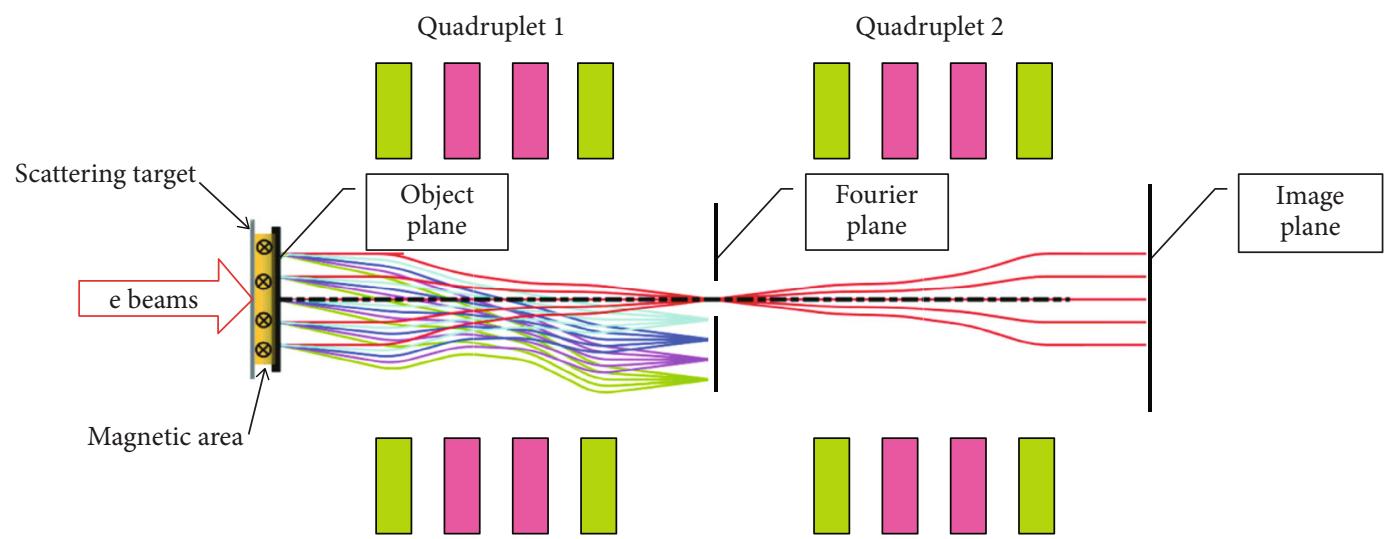

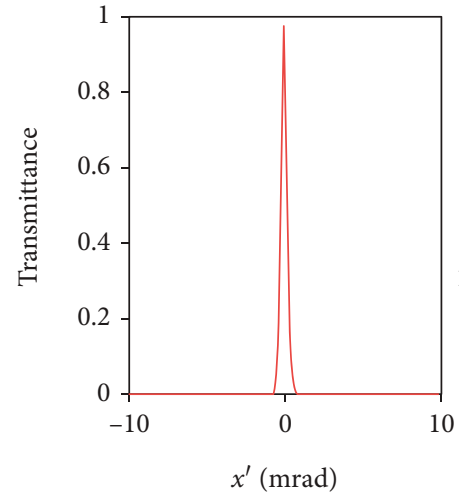

(a)

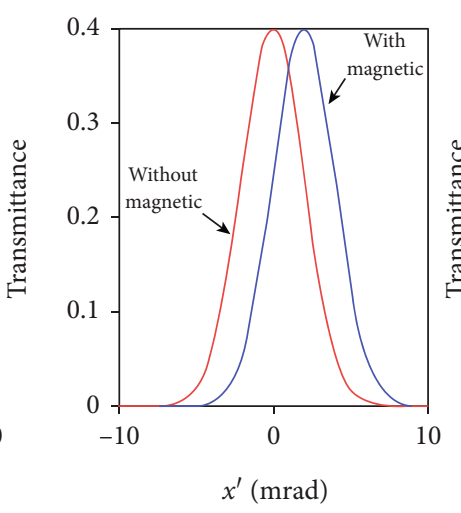

(b)

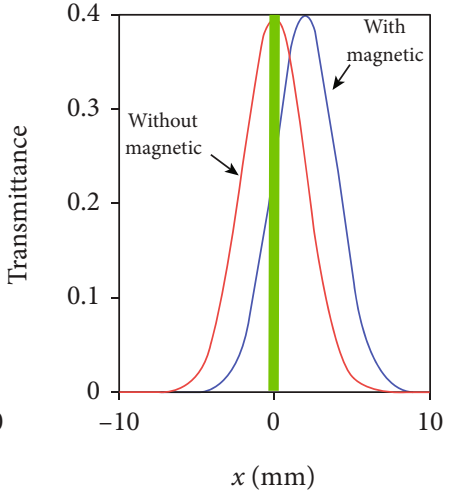

(c)

FIgURE 1: (color online). Schematic diagram of HEER for magnetic area diagnosis. (a) The angle distribution of incident electrons. (b) The electrons angle distribution after scattering target. (c) The electron position at Fourier plane; the green shadow area is the aperture position.

The whole diagnosis process is divided into two parts to describe the interaction of beam line with plasma and the electrons transport in the point-to-point HEER beam line.

For the first subprocess, high-energy electron beams will be deflected by a magnetic field when penetrating the system. The deflection can be described by the following formula:

$$
\theta=\int \omega d t=\int \frac{e B_{\perp} d t}{\gamma m_{0}}=\int \frac{e B_{\perp}}{\gamma m_{0} c} d z
$$

where

$$
\gamma=\frac{1}{\sqrt{1-\left(\mathrm{v}^{2} / \mathrm{c}^{2}\right)}}
$$

where $\theta$ is deflection of electron beam, $\omega$ is angular velocity of electrons in magnetic field, $\mathrm{v}$ is velocity of electrons, $m_{0}$ is the electron rest mass, $c$ is speed of light in vacuum. $B_{\perp}$ is the weight of magnetic vector at $x-y$ plane, and $z$ is the position of magnetic area.

Based on the above description, if the incident electrons have a specific angle distribution with high symmetry in polar coordinate at $x-y$ plane, there is one-to-one correspondence between transmittance and deflection.
The angle distribution of monoenergtetic electron interacting with matter can be described by the following function:

$$
f(\phi)=\frac{N(t, \phi)}{N_{0}}=\frac{1}{\phi_{0}(t) \sqrt{2 \pi}} e^{-(1 / 2)\left(\phi / \phi_{0}(t)\right)^{2}},
$$

where

$$
\phi_{0}(t)=\frac{13.6 \mathrm{MeV}}{\beta c p} \sqrt{t}(1+0.038 \ln (t)),
$$

where $\phi$ is scattered angle of electrons, $\mathrm{t}=X / X_{0}, X$ is the thickness of target, and $X_{0}$ is the radio length of specimen materials.

From formula (3), after interactions between the incident electron and target, the electron beams will have a specific angle distribution. And the distribution should have high symmetry in polar coordinate. In other words, the angle distribution of incident electrons can be controlled by scattering target so as to meet diagnostic demand.

The next step is deflection measured by HEER beam line. For monoenergetic electron beams, the transport in beam line can be explained by the following matrix: 


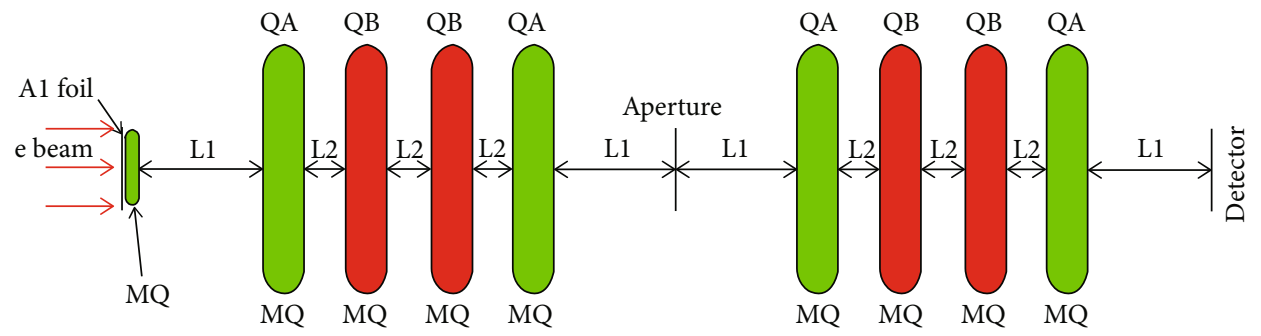

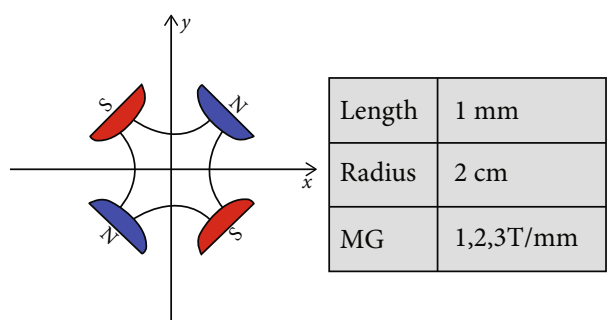

(a)

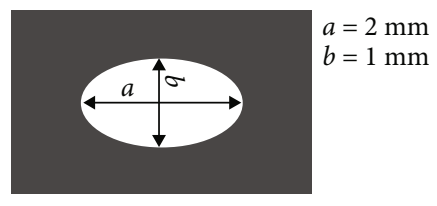

(b)

Figure 2: (color online). Schematic of simulation design. The magnetic area as the specimen is a quadrupole magnet (a). And the aperture is designed as an ellipse, where $x$-half-axis is $1 \mathrm{~mm}$ and $y$-half-axis is $0.5 \mathrm{~mm}$ in length (b).

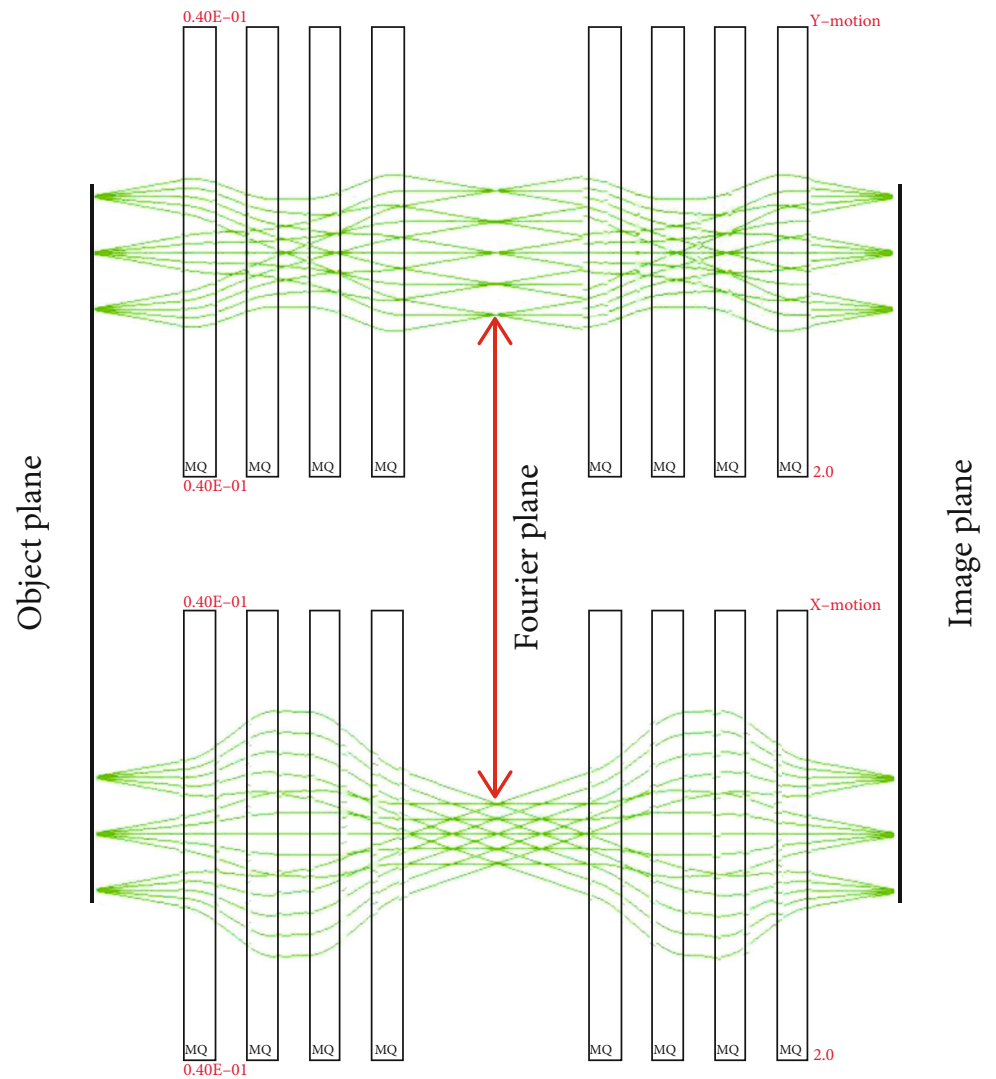

FIGURE 3: Electron beam trace from object plane to image plane in the $x$-plane and $y$-plane. 
TABLE 1: The transport matrix parameters of radiography beam line.

\begin{tabular}{lccr}
\hline Parameters & Value & Parameters & Value \\
\hline $\mathrm{R}_{11 \mathrm{~F}}$ & $-2.69 \times 10^{-3}$ & $R_{12 \mathrm{~F}}$ & $1.10 \mathrm{~mm} / \mathrm{mrad}$ \\
$\mathrm{R}_{33 \mathrm{~F}}$ & $-6.20 \times 10^{-3}$ & $\mathrm{R}_{34 \mathrm{~F}}$ & $0.53 \mathrm{~mm} / \mathrm{mrad}$ \\
$\mathrm{R}_{11}$ & -1.00 & $\mathrm{R}_{12}$ & $-5.94 \times 10^{-3} \mathrm{~mm} / \mathrm{mrad}$ \\
$\mathrm{R}_{33}$ & -1.00 & $\mathrm{R}_{34}$ & $-6.56 \times 10^{-3} \mathrm{~mm} / \mathrm{mrad}$ \\
\hline
\end{tabular}

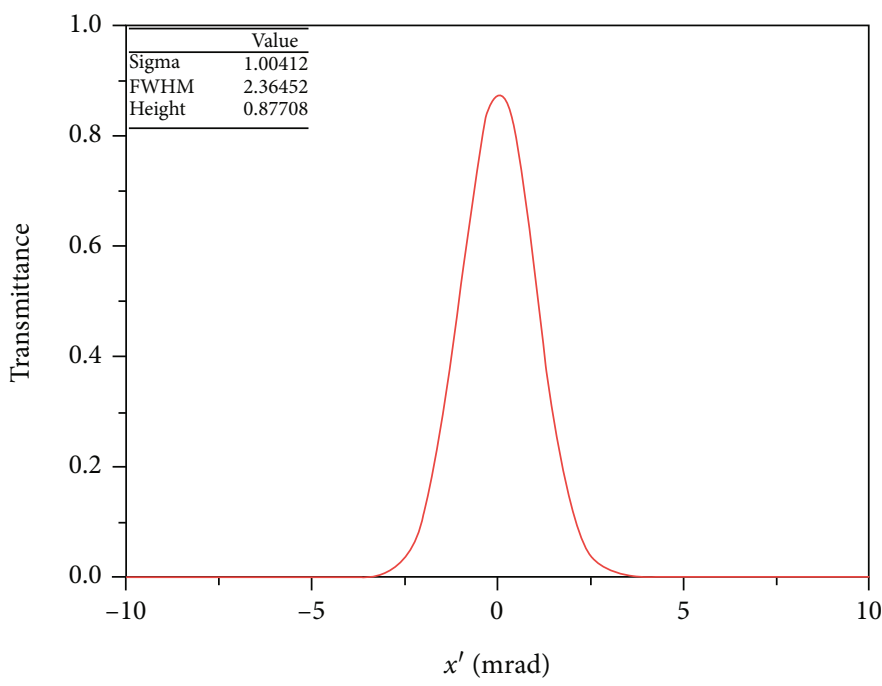

FIGURE 4: Angle distribution of scattered electrons.

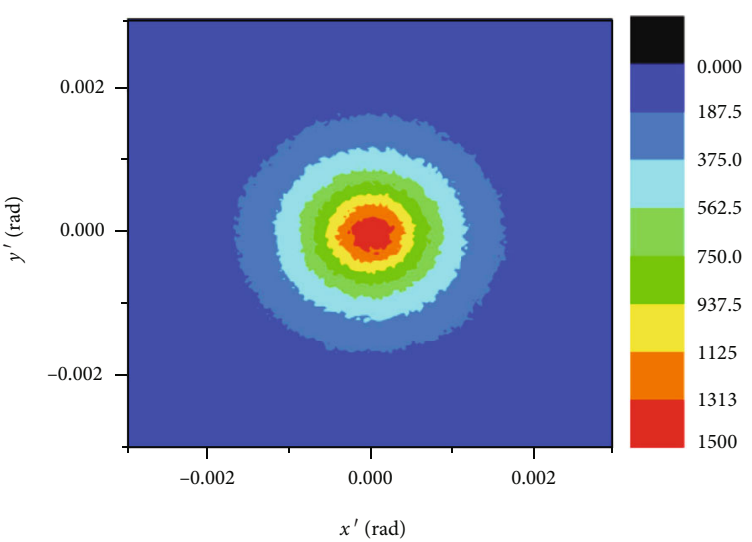

(a)

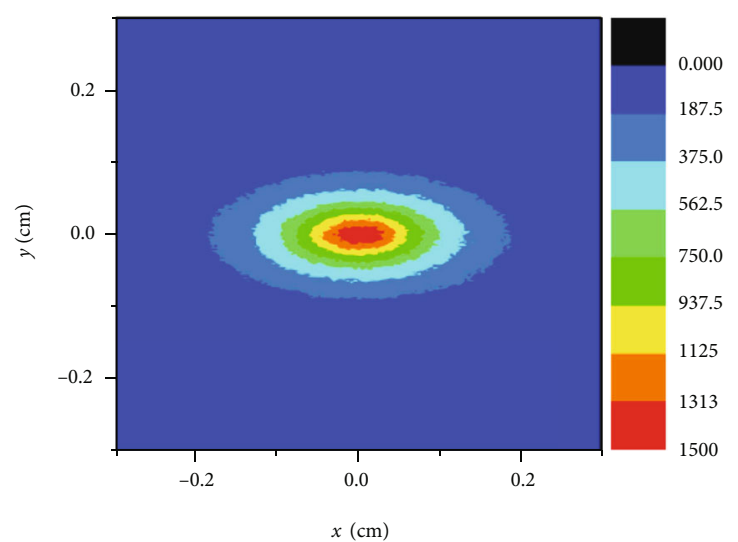

(b)

Figure 5: (color online). The angle distribution of electrons after scattering target (a) and the position distribution of electrons at the Fourier plane (b).

$$
\left[\begin{array}{c}
x_{i} \\
x_{i}^{\prime} \\
y_{i} \\
y_{i}^{\prime}
\end{array}\right]=\left[\begin{array}{cccc}
R_{11} & R_{12} & 0 & 0 \\
R_{21} & R_{22} & 0 & 0 \\
0 & 0 & R_{33} & R_{34} \\
0 & 0 & R_{43} & R_{44}
\end{array}\right]\left[\begin{array}{c}
x_{0} \\
x_{0}^{\prime} \\
y_{0} \\
y_{0}^{\prime}
\end{array}\right],
$$

where $x_{i}, y_{i}$ and $x_{i}^{\prime}, y_{i}^{\prime}$ are the position and angle of electrons at the observation plane, and $R_{i j}$ are the conversion factor of the beam line. The $x_{0}, y_{0}$ and $x_{0}^{\prime}, y_{0}^{\prime}$ are the position and angle of electrons at the emission plane [27].

At the image plane, transport matrix parameters $R_{12}=$ $R_{34}=0$. It means that the electron position at the image plane is irrelevant with the angle of electrons at the object plane. This beam line design is called point-to-point radiography. Between the image plane and the object plane, by optimizing, there will be a plane named Fourier plane which matches the transport matrix parameters $R_{11}=R_{33}=0$. It means that 

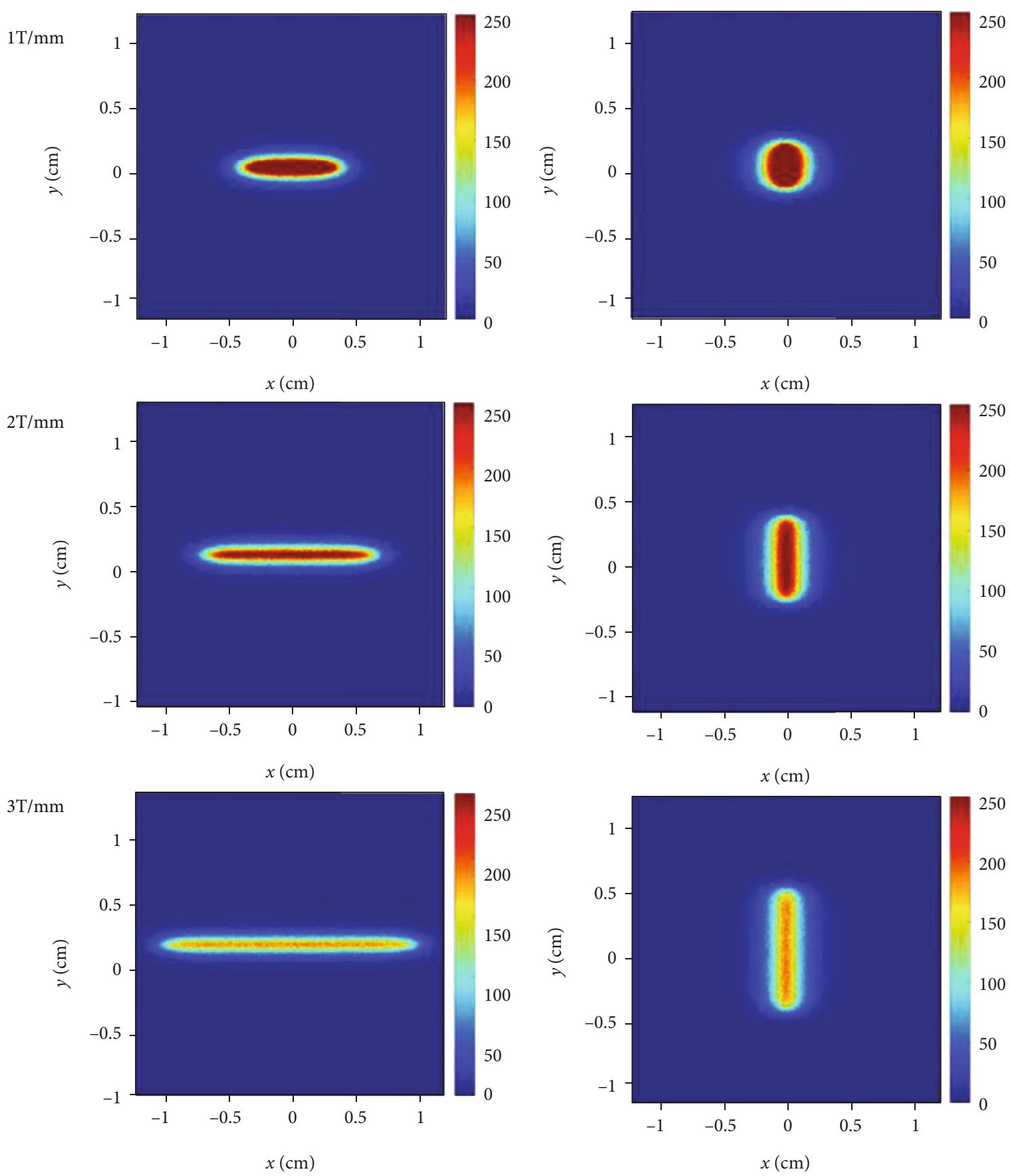

(a) The incident line beam is along $x$-axis

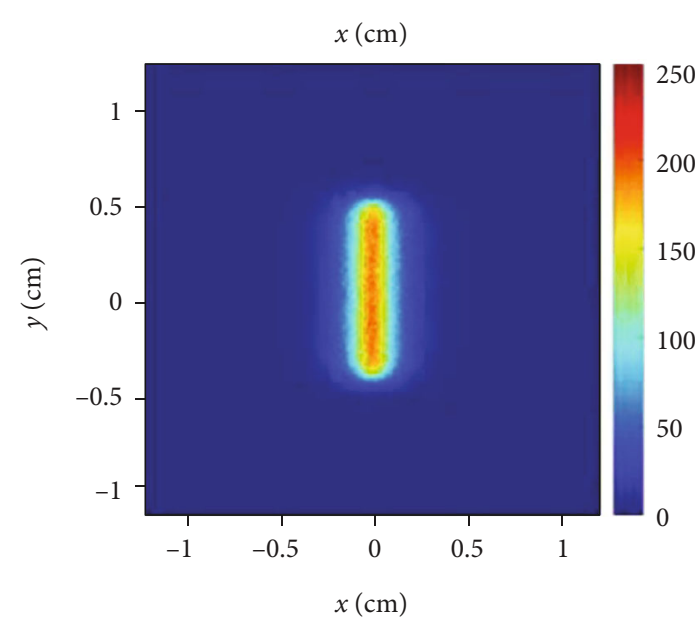

(b) The incident line beam is along $y$-axis

FIgURE 6: The electron position distribution at the Fourier plane under different specimen quadrupole designs.

the electron angle information at object plane has been translated to position information at this plane. Thence, an aperture at the Fourier plane can achieve angle selection for transmitted electrons. In the past years, the application of aperture is to get better spatial and areal density resolutions. Now, the magnetic strength diagnosis depends on the characteristics of the aperture.

The aperture should be set at the Fourier plane where aperture can select the angle around the center axis when the electrons are not deflected. If the electron beam collective is deflected by the magnetic field, the angle selection will also be deflected from the center axis of angle distribution. Thence, for the incident electrons with specific angle distribution, the deflection of them has a negative correlation with its transmittance. Furthermore, it is necessary to make the ratio of the $x$ -half-axis and $y$-half-axis equal $R_{12 \mathrm{~F}} / R_{34 \mathrm{~F}}$ to ensure one-toone correspondence between magnetic strength and transmittance. The footnote $F$ means that the transport matrix parameters are from the object plane to Fourier plane. The principle is shown in Figure 1. 

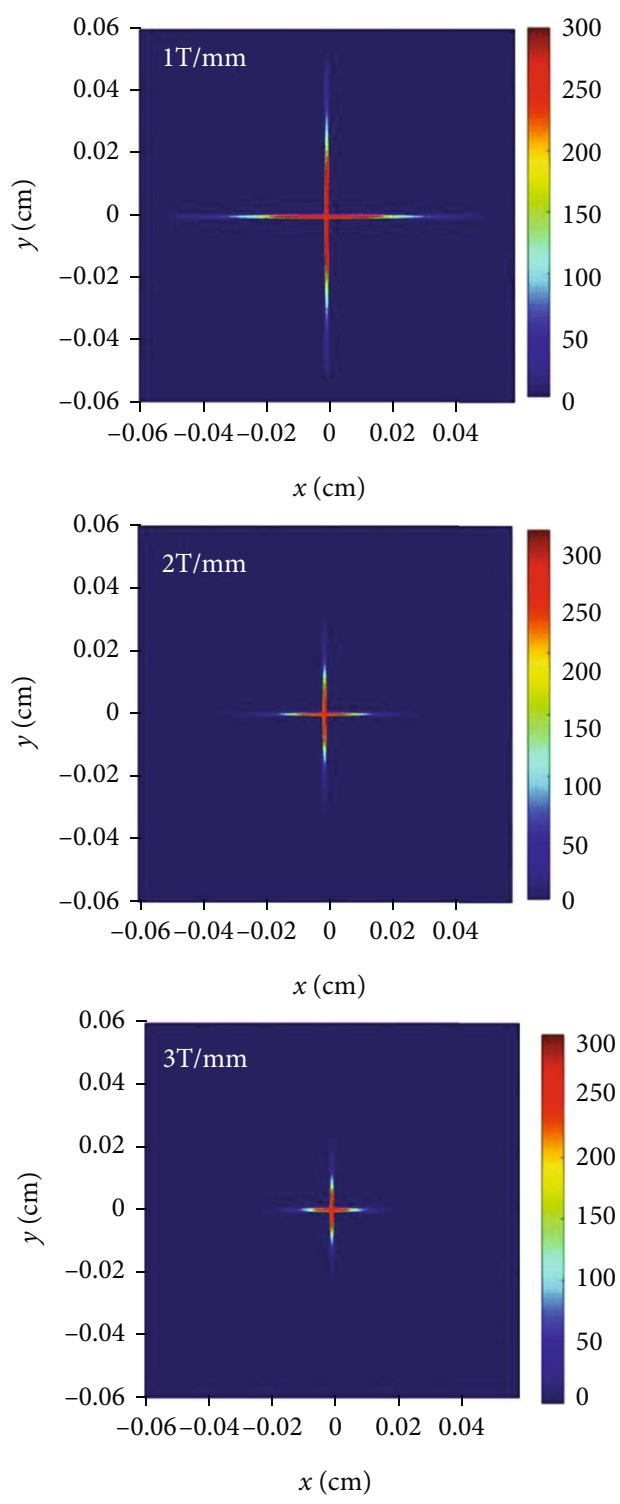

(a)
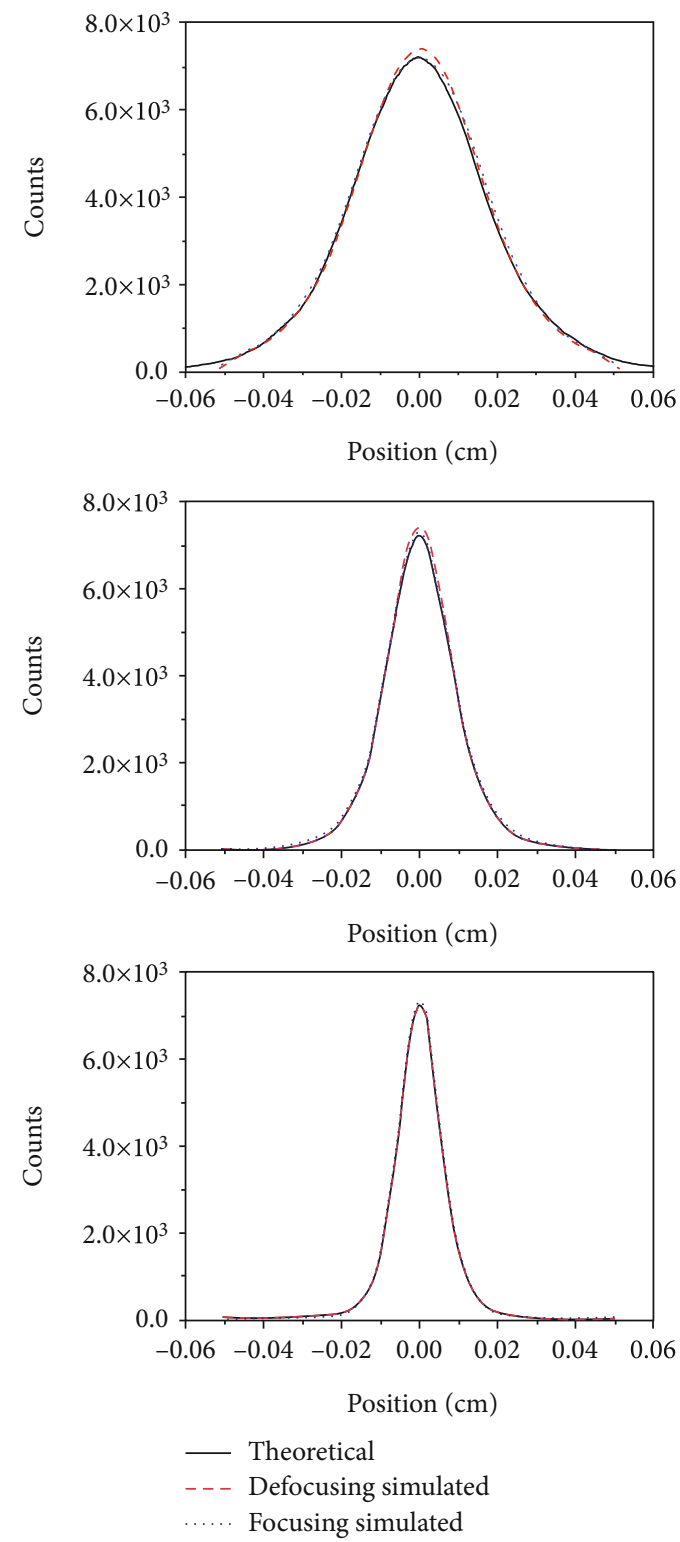

(b)

Figure 7: (color online). Simulation results under different designs for quadrupole magnetic field as specimens.

\section{Simulations}

3.1. General Setup. The sketch of integral simulation is shown in Figure 2. The incident electrons are scattered by an aluminum foil initially, and then, the scattered electrons penetrate the magnetic vacuum. Then, HEER magnetic lens are set to transport, select, and refocus the electrons behind target. Finally, the electron number density will be counted by the detector at image plane.

3.2. Image Lens Optimization. The typical charged particle image beam line should satisfy that the transport matrix parameters are $R_{12}=R_{34}=0$ at the image plane. But for the force field diagnosis HEER, as mentioned in Section 2, it is necessary to make the $x$-axis and $y$-axis Fourier plane coincident run along the $z$-axis. Under this requirement, the imaging lens system adapting $50 \mathrm{MeV}$ kinetic energy electron beams is optimized by COSY INFINITY 9.1 [28]. It includes eight quadrupole magnets, as shown in Figure 2; the length of them is $10 \mathrm{~cm}$, the inner diameter is $40 \mathrm{~mm}$, and the MG of QA is $3.9097 \mathrm{~T} / \mathrm{m}$, and QB is $-3.6295 \mathrm{~T} / \mathrm{m}$. The drift length $\mathrm{L} 1$ is $30 \mathrm{~cm}$, and $\mathrm{L} 2$ is $10 \mathrm{~cm}$. The total length of this beam line is $2.6 \mathrm{~m}$. The optimization result is shown in Figure 3. And the relational transport matrix parameters of it are shown in Table 1.

According to transport matrix parameters from the object plane to Fourier plane, the aperture in the simulation is designed as an ellipse shape as shown in Figure 2(b) and 


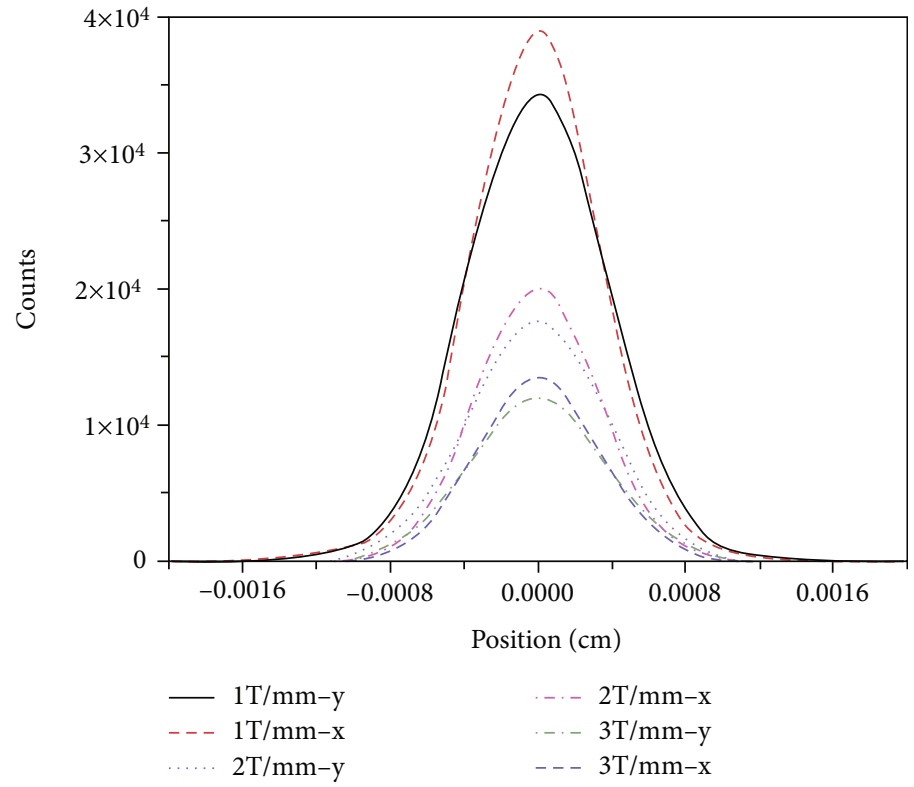

Figure 8: (color online). The distribution of electrons at image plane in different specimen quadrupole designs.

the corresponding selection angle range can be described by the following formula:

$$
x_{0}^{\prime 2}+y_{0}^{\prime 2} \leq(0.91 \mathrm{mrad})^{2} .
$$

3.3. Specimen Design. The specimen is composed of scattering target and magnetic vacuum. In this work, we select a $5 \mu \mathrm{m}$ thick aluminum foil as the scattering target. On the one hand, as a low $Z$ element, it can ensure that the probe electrons have lower energy spread. On the other hand, aluminum is always selected as the target material in the research of intense laser-solid interaction or $Z$-pinch. Combined with the aperture selection, the transmittance corresponding to deflection is shown in Figure 4.

For this Gaussian distribution, we delimit the transmittance larger than 0.01 as detectable. That means the detection range of deflection is from $-3 \mathrm{mrad}$ to $3 \mathrm{mrad}$. For $50 \mathrm{MeV}$ electrons, the corresponding magnetic strength diagnostic range is from $-0.51 \mathrm{~T}^{*} \mathrm{~mm}$ to $0.51 \mathrm{~T}^{*} \mathrm{~mm}$.

Except for the lens design, there are two factors that might influence measuring accuracy in this design: deflection and magnetic gradient (MG) of the specimen. The deflection can be limited by the incident electron energy. To get microspatial resolution, we should control the deflection less than $10 \mathrm{mrad}$ for $1 \mathrm{~mm}$ scale expansion size. To study the influence of MG on measurement accuracy, we select a quadrupole model as the magnetic vacuum in this simulation, and a group of MG is set as 1,2 , and $3 \mathrm{~T} / \mathrm{mm}$.

3.4. Complete Simulation Process. We simulate the situation that the line beam along the $x$-axis and $y$-axis penetrates the specimen. This set has two advantages. Firstly, the focusing and defocusing influences can be analyzed, respectively; secondly, the spatial resolution along the $x$-axis and $y$-axis also can be self-governed. The whole simulation of diagnostic process includes three sections: incident electrons interact
TABLE 2: The RMS spatial resolution along the $x$-axis and $y$-axis.

\begin{tabular}{lcc}
\hline MG & RMS $(x)$ & RMS $(y)$ \\
\hline $1 \mathrm{~T} / \mathrm{mm}$ & $3.4 \mu \mathrm{m}$ & $3.7 \mu \mathrm{m}$ \\
$2 \mathrm{~T} / \mathrm{mm}$ & $3.4 \mu \mathrm{m}$ & $3.7 \mu \mathrm{m}$ \\
$3 \mathrm{~T} / \mathrm{mm}$ & $3.4 \mu \mathrm{m}$ & $3.7 \mu \mathrm{m}$ \\
\hline
\end{tabular}

with scattering target, electron beams go through the area with electromagnetic field, and electrons are refocused by HEER beam line. For the first simulation section, EGS5 codes are selected to do this [29]. Because the magnetic field model is the standard quadrupole field as the specimen, the last two section simulation is done by PARMELA software [30].

\section{Results and Discussion}

In the case of specimen with no magnetic vacuum, the scattered electron angle distribution and position distribution at Fourier plane are counted. The statistical steps of angle distribution are $0.04 \mathrm{mrad}$ along $x^{\prime}$ and $y^{\prime}$ and of position are $0.044 \mathrm{~mm}$ along the $x$-axis and $0.021 \mathrm{~mm}$ along $y$-axis, respectively. The results are shown in Figure 5.

According to Figure 5, we verify that the angle distribution after scattering target is translated to position distribution at Fourier plane and the convert ratios correspond to $R_{12 \mathrm{~F}}$ and $R_{34 \mathrm{~F}}$ along the $x$-axis and $y$-axis, respectively.

Figure 6 shows the electron position distribution at the Fourier plane when different magnetic field gradients of specimen magnetic are set. Compared with Figure 5, the electron distribution at the Fourier plane is deformed. The distribution is broadened along the direction of Lorentz force in the magnetic field. The size of line beam broadening is related with the magnetic gradient. In this case, the transmittance will be also different when the same aperture is used in the simulation. 
We integrate the two line beams into a crossing beam as a probe electron beam, and the diagnosis results for the quadrupole magnetic field with different magnetic gradients are shown in Figure 7. Based on the distribution of incident angle distribution, the range of diagnosis is from $-0.51 \mathrm{~T}^{*} \mathrm{~mm}$ to $0.51 \mathrm{~T}^{*} \mathrm{~mm}$. Corresponding to different magnetic gradient settings, the range is from $-0.51 \mathrm{~mm}$ to approximately $0.51 \mathrm{~mm}, \quad-0.26 \mathrm{~mm}$ to approximately $0.26 \mathrm{~mm}$, and $-0.17 \mathrm{~mm}$ to approximately $0.17 \mathrm{~mm}$. In Figure 7 , the simulation results are very consistent with the theoretical value regardless of the magnetic focusing or defocusing interactions and a little deviation in the measurement at the weak field area. That means, at the edge of the magnetic area, there might be deviation in the experiment.

The spatial resolution can be taken by the analysis of the line beam broadening at the image plane. The statistical result of electron distribution at image plane is shown in Figure 8.

And the following analysis results are shown in Table 2.

The simulation results indicate that the RMS spatial resolution of this design is better than 4 micrometers along the $x$-axis and $y$-axis. And the distribution of the magnetic strength field taken by this method is continuous. In fact, for the smaller size specimen, the spatial resolution can be also improved by cascade magnification HEER so as to get more delicate structure.

\section{Conclusions}

We present a new method to diagnose the transient magnetic field that is based on the HEER beam line design. The simulation results of $50 \mathrm{MeV}$ penetrating the quadrupole model indicates that this method has high accuracy for strong field measurement no matter whether the magnetic gradient interaction is focusing or defocusing. At the same time, spatial resolution better than 4 micrometers is also got in our simulations. Of course, smaller aperture application or more optimized beam line design can get better spatial resolution. In summary, these results indicate that this method is very suitable for the delicate diagnosis of the transient magnetic field strength.

\section{Data Availability}

The data used to support the findings of this study are available from the corresponding author upon request.

\section{Conflicts of Interest}

The authors declare that there is no conflict of interest regarding the publication of this paper.

\section{Acknowledgments}

This work is supported by the National Key R\&D Program of China under Grant (No. SQ2019YFA040016).

\section{References}

[1] M. G. Haines, "Saturation mechanisms for the generated magnetic field in nonuniform laser-matter irradiation," Physical Review Letters, vol. 78, no. 2, pp. 254-257, 1997.

[2] L. Spitzer, Physics of fully ionized gases, Interscience, New York, 1962.

[3] S. I. Braginskii, Review of plasma physics, Consultant Bureau, New York, 1965.

[4] X. Y. Zhao, L. Y. Zhang, Y. L. Zhang et al., "The influence of magnetic fields on the wake field and stopping power of an ion-beam pulse in plasmas," Physics of Plasmas, vol. 22, article $093114,2015$.

[5] N. S. Edison, B. Etlicher, A. S. Chuvatin, S. Attelan, and R. Aliaga, "Enhanced Z pinch using an externally applied magnetic field to stabilize the implosion of an aluminum plasma jet onto a coaxial wire," Physical Review E, vol. 48, pp. 3893-3900, 1993.

[6] J. Brynolf, R. Ring, and C. Wahlberg, "MHD spectrum of a Zpinch in an external magnetic multipole field," Plasma Physics and Controlled Fusion, vol. 27, no. 11, pp. 1255-1283, 1985.

[7] T. Tajima and J. M. Dawson, "Laser electron accelerator," Physical Review Letters, vol. 43, no. 4, pp. 267-270, 1979.

[8] P. Chen, J. M. Dawson, R. W. Huff, and T. Katsouleas, “Acceleration of electrons by the interaction of a bunched electron beam with a plasma," Physical Review Letters, vol. 54, pp. 693-696, 1985.

[9] A. Pukhov, Z. M. Sheng, and J. Meyer-ter-Vehn, "Particle acceleration in relativistic laser channels," Physics of Plasmas, vol. 6, no. 7, pp. 2847-2854, 1999.

[10] T. R. Dittrich, S. W. Haan, M. M. Marinak et al., "Review of indirect-drive ignition design options for the National Ignition Facility," Physics of Plasmas, vol. 6, no. 5, pp. 2164-2170, 1999.

[11] S. Eliezer, The Interaction of High-Power Lasers with Plasmas, CRC Press, Boca Raton, FL, 2002.

[12] A. Ng, T. Ao, F. Perrot, M. W. Dharma-Wardana, and M. E. Foord, "Idealized slab plasma approach for the study of warm dense matter," Laser Particle Beams, vol. 23, no. 4, pp. 527537, 2005.

[13] W. Schumaker, N. Nakanii, C. McGuffey et al., "Ultrafast electron radiography of magnetic fields in high-intensity lasersolid interactions," Physical Review Letters, vol. 110, article 015003, 2013.

[14] C. K. Li, F. H. Séguin, J. A. Frenje et al., "Proton radiography of dynamic electric and magnetic fields in laser-produced highenergy-density plasmas," Physics of Plasmas, vol. 16, article 056304, 2009.

[15] C. K. Li, F. H. Séguin, J. A. Frenje et al., "Measuring E and B fields in laser-produced plasmas with monoenergetic proton radiography," Physical Review Letters, vol. 97, article 135003, 2006.

[16] L. Willingale, P. M. Nilson, M. C. Kaluza et al., "Proton deflectometry of a magnetic reconnection geometry," Physics of Plasmas, vol. 17, article 043104, 2010.

[17] K. Quinn, P. A. Wilson, C. A. Cecchetti et al., "Laser-driven ultrafast field propagation on solid surfaces," Physical Review Letters, vol. 102, no. 19, p. 194801, 2009.

[18] P. F. Zhu, Z. C. Zhang, L. Chen et al., "Ultrashort electron pulses as a four-dimensional diagnosis of plasma dynamics," The Review of scientific instruments, vol. 81, article 103505, 2010. 
[19] L. Chen, R. Z. Li, J. Chen et al., "Mapping transient electric fields with picosecond electron bunches," Proceedings of the National Academy of Sciences, vol. 112, article 14479, 2015.

[20] F. Merrill, F. Harmon, A. Hunt et al., "Electron radiography," Nuclear Instruments and Methods in Physics Research Section B: Beam Interactions with Materials and Atoms, vol. 26, pp. 382-386, 2007.

[21] Q. Zhao, S. C. Cao, M. Liu et al., "High energy electron radiography system design and simulation study of beam angleposition correlation and aperture effect on the images," Nuclear Instruments \& Methods in Physics Research. Section A, vol. 832, pp. 144-151, 2016.

[22] Y. Zhao, Z. Zhang, W. Gai et al., "High energy electron radiography scheme with high spatial and temporal resolution in three dimension based on a e-linac," Laser and Particle Beams, vol. 34, no. 2, pp. 338-342, 2016.

[23] J. Xiao, Z. Zhang, S. Cao et al., "Areal density and spatial resolution of high energy electron radiography," Chinese Physics $B$, vol. 27, article 035202, 2018.

[24] F. E. Merrill, J. Goett, J. W. Gibbs et al., "Demonstration of transmission high energy electron microscopy," Applied Physics Letters, vol. 112, article 144103, 2018.

[25] Z. Zhou, Y. Du, S. Cao et al., "Experiments on bright field and dark field high energy electron imaging with thick target material," Physical Review Accelerators and Beams, vol. 21, article 074701, 2018.

[26] Z. Zhou, Y. Fang, H. Chen et al., "Demonstration of singleshot high-quality cascaded high-energy-electron radiography using compact imaging lenses based on permanent-magnet quadrupoles," Physical Review Applied, vol. 11, article 034068, 2019.

[27] K. L. Brown, "A first- and second-order matrix theory for the design of beam transport systems and charged particle spectrometers," Stanford Linear Accelerator Center, California, 1972.

[28] M. Berz and K. Makino, COSY INFINITY 9.1 Beam Physics Manual, MSU Report MSUHEP 060804-Rev, MSU, East Lansing, MI, USA, 2013.

[29] H. Hirayama, Y. Namito, A. F. Bielajew, S. J. Wilderman, and W. R. Nelson, The EGS5 Code System, 2005, SLAC-R-730 (2005) and KEK Report 2005-8.

[30] L. M. Young, "PARMELA," Los Alamos National Laboratory report LA-UR-96-1835, 2005. 